\title{
Elucidating the Functional Relationship Between Speed of Information Processing and Speed-, Capacity-, and Memory-Related Aspects of Psychometric Intelligence
}

\author{
Olivier Pahud' ', Thomas H. Rammsayer', and Stefan J. Troche ${ }^{2}$ \\ ${ }^{1}$ Institute of Psychology, University of Bern, Fabrikstrasse 8, CH-3012 Bern, Switzerland \\ ${ }^{2}$ Department of Psychology and Psychotherapy, University of Witten/Herdecke, \\ Alfred-Herrhausen-Straße 50, D-58448 Witten, Germany
}

ABSTRACT

\begin{abstract}
Numerous studies reported a positive relationship between speed of information processing (SIP) and the $g$ factor of intelligence. Only very few studies, however, examined SIP's relationship to speed-, capacity-, and memory-related aspects of psychometric intelligence. In order to further elucidate this relationship, a Hick reaction time task and the Berlin Intelligence Structure (BIS) test were administered to 240 participants. From the BIS test, indicators of BIS-Capacity, BIS-Speed, and BIS-Memory were determined. By means of fixed-links modeling, we subdivided variance in reaction time from the Hick task into a component representing individual differences in speed directly related to the systematically increased number of possible responses and another component representing individual differences in speed associated with residual sources unrelated to the experimental variation of response alternatives. While the former speed component was primarily related to BIS-Capacity and, to a lesser extent, to BIS-Speed, the latter one was only weakly related to BIS-Speed but unrelated to BIS-Capacity. None of the two speed components from the Hick task showed an association with BIS-Memory. Our findings indicate that individual differences in SIP caused by experimentally increasing the number of possible responses were more strongly associated with capacity- than with speed-related aspects of psychometric intelligence. In addition, individual differences in SIP not associated with the experimental manipulation of response alternatives showed rather weak relationships to each of the three BIS operations and could be considered equally strong.
\end{abstract}

\section{reaction time, mental ability, Hick paradigm, mental speed approach, fixed-links modeling, Berlin Intelligence Structure Test \\ KEYWORDS}

\section{INTRODUCTION}

A large number of studies provide empirical evidence for the notion that individual differences in speed of information processing (SIP) are associated with individual differences in psychometric intelligence (for reviews see Jensen, 1998a, 2006; Sheppard \& Vernon, 2008). In other words, individuals with higher mental ability show faster reaction times in tasks measuring simple cognitive processes. One of the most frequently used tasks to assess SIP represents the reaction time paradigm following the rationale of Hick (1952). The Hick paradigm is a visual simple and choice reaction time task, in which participants have to respond as quickly as possible to an upcoming stimulus. In the case of simple reaction time, no decision between response alternatives is involved, and thus, zero bits of information have to be processed. Therefore, this condition is referred to as 0-bit condition. Analogously, deciding between two response alternatives, as required by a two-

Corresponding author: Olivier Pahud, University of Bern, Institute of Psychology, Fabrikstrasse 8, CH-3012 Bern, Phone: +41 (0)31/631'47'27. E-mail: olivier.pahud@psy.unibe.ch 
choice reaction time task, necessitates one binary decision (1-bit condition). When four response alternatives are present, as in the case of a typical four-choice reaction time task, two binary decisions are necessary (2-bit condition). Hick discovered a linear relationship between an individual's reaction time and the number of bits of information to be processed (i.e., the $\log 2$ of the number of response alternatives).

The vast majority of studies within the mental speed approach to intelligence related SIP to global measures of psychometric intelligence, such as full-scale IQ or general intelligence as indicated by psychometric $g$ (cf. Jensen, 1987, 2006; Sheppard \& Vernon, 2008). As an exception to this, Beauducel and Brocke (1993) related Hick reaction time parameters to more specific aspects of psychometric intelligence as measured with the Berlin Intelligence Structure (BIS) Test (Jäger, Süss, \& Beauducel, 1997), namely processing speed (BIS-Speed), processing capacity (BIS-Capacity), and memory (BIS-Memory). Hick reaction times showed moderate correlations with BIS-Speed, but weak correlations with BIS-Capacity and BIS-Memory. Albeit reporting stronger associations than Beauducel and Brocke, a similar correlational precedence was reported by Neubauer and Bucik (1996). The correlations reported by these two studies suggest that SIP is primarily related to the processing speed aspect and, to a lesser extent, to the capacity and memory aspect of intelligence.

However, this conclusion has to be taken with caution for several reasons. First, Beauducel and Brocke (1993) used the Hick task with a manifest-variable approach, whereas Neubauer and Bucik (1996) used a latent-variable approach with several different paper-andpencil SIP measures. Manifest approaches directly rely on observed variables, which are subject to measurement error. In contrast, latent approaches account for measurement error by explicitly representing error estimates in a model, and thus, latent variables comprise true variance shared by several observed variables (Kline, 2011). Therefore, the two methodological strategies led to different operationalizations of SIP. Second, due to the fact that Neubauer and Bucik solely used paper-and-pencil measures, the correlation between SIP and psychometric processing speed could be overestimated because of shared method-specific variance. Third, research on mental chronometry has raised awareness of the fact that reaction time on any cognitive task is a composite measure of the time taken by a number of different cognitive processes involved in the completion of a given task (e.g., Jensen, 1987; Luce, 1986; Miller \& Ulrich, 2013; Unsworth \& Engle, 2007; Van Zomeren \& Brouwer, 1994). Additionally, individual state-dependent factors, such as levels of motivation or alertness, can also influence response latency (Humphreys \& Revelle, 1984; Langner, Steinborn, Chatterjee, Sturm, \& Willmes, 2010; Lisper \& Kjellberg, 1972). Hence, the interpretation of the correlational relationship between SIP and psychometric intelligence is blurred, as it is not clear to what extent different sources of variance account for the observed correlation. In the realm of individual differences, Schweizer (2007) referred to this confounding effect of different sources of variance as the impurity problem.

For the Hick paradigm, different approaches were developed to control for the impurity problem. For example, modifications of the experimental design were introduced to minimize residual sources of variance by controlling for movement times (Jensen \& Munro, 1979) or stimulus-response compatibility (Alluisi \& Warm, 1990). In addition, various measures were used to account for different sources of reaction time variance (Jensen, 1987). In particular, the individual slope of reaction time across the different Hick conditions and the intercept were determined using regression analysis. The former has been suggested to represent a speed measure of the experimentally manipulated process, that is, the time needed for a single binary decision, whereas the latter has been considered to reflect the residual sources of reaction time (cf. Jensen, 1987, 1998b; Sternberg, 1969). Jensen (1998b), however, showed that disentangling different aspects of SIP by means of regression-based slope and intercept bears severe methodological problems. Above all, the possible negative correlation between shared errors of measurement of slope and intercept can cause a suppression effect leading to an underestimation of the true relationship between these parameters and any third variable. Hence, if one of these two parameters is correlated with psychometric intelligence, the other parameter might act as a suppressor variable on that correlation resulting in a markedly reduced correlational relationship (e.g., Bors \& Forrin, 1995; Jensen 1987, 1998b).

Previously, Schweizer $(2006,2008)$ introduced fixed-links modeling as an alternative methodological approach to cope with the impurity problem. Fixed-links modeling is a special form of confirmatory factor analysis for experimental repeated-measures designs. With this approach, overall reaction time variance is decomposed into two components: an experimental and a nonexperimental latent variable. The experimental latent variable represents individual differences in reaction time due to the specific manipulation of the experimental task. In case of the Hick reaction time paradigm, on the one hand, this is the variance in reaction time as a function of the increasing number of response alternatives. On the other hand, the nonexperimental latent variable represents individual differences in the residual sources of reaction time that are considered constant across task conditions, and thus, unaffected by the systematic experimental manipulation (cf. Schweizer, 2007, 2008). This latent variable includes, for example, general (i.e., task-independent) SIP such as perceptual and motor speed (Schweizer, 2007; Stauffer, Troche, Schweizer, \& Rammsayer, 2014) or an individual's general state of alertness, fatigue, or motivation (Thomas, Rammsayer, Schweizer, \& Troche, 2015). In contrast to the slope-intercept approach, the fixed-links approach is not affected by suppression effects, since the correlation between the experimental and the nonexperimental latent variable is explicitly set to zero. Such a correlation between two predictor variables, however, is a necessary condition for a suppression effect (Tabachnick \& Fidell, 2013).

In order to extract these two latent variables from the same set of manifest variables, fixation of factor loadings is required. The factor loadings of the experimental latent variable are fixed in accordance with a theoretically expected trajectory caused by the experimental manipulation (e.g., an increasing trajectory across task conditions), whereas all factor loadings of the nonexperimental latent variable are fixed to the same value (e.g., 1) indicating consistency across task conditions. 
Given that all factor loadings are fixed, variance of the experimental as well as the nonexperimental latent variable is set free for estimation and needs to reach statistical significance in order to be interpreted as psychologically meaningful. Fixed-links modeling has been successfully applied to decompose experimental from nonexperimental variance for various cognitive processes such as working memory (Schweizer, 2007; Thomas et al., 2015), visual change detection (Stauffer et al., 2014), and attention (Ren, Schweizer, \& Xu, 2013; Troche, Schweizer, \& Rammsayer, 2009; Wagner, Rammsayer, Schweizer, \& Troche, 2014, 2015). In a previous study, Rammsayer, Pahud, and Troche (2017) successfully dissociated Hick reaction time variance into an experimental as well as a nonexperimental latent variable and related them to $\mathrm{g}$. This study showed that the relationship between SIP and psychometric $g$ is primarily driven by individual differences in reaction time influenced by the experimental manipulation of response alternatives, whereas individual differences in reaction time represented by the nonexperimental component only explained a marginal portion of variance in $\mathrm{g}$.

Against this background, and as an extension of our previous study (Rammsayer et al., 2017), the major goal of the present study was to arrive at a better understanding of the relationship between SIP and speed-, capacity-, and memory-related aspects of psychometric intelligence. For this purpose, we examined the relationship between mean Hick reaction times and these three major components of intelligence with a manifest approach as in Beauducel and Brocke (1993), as well as on a latent level as in Neubauer and Bucik (1996). Additionally, we applied fixed-links modeling to take care of impure SIP by decomposing Hick reaction time variance into an experimental and a nonexperimental source. The former one was supposed to represent Hick-specific reaction time variance that was directly related to the increase in response alternatives across task conditions, whereas the latter one was supposed to represent task-independent reaction time variance constant across task conditions. In particular, we analyzed to what degree these dissociated portions of reaction time variance predict speed-, capacity-, and memory-related aspects of psychometric intelligence.

\section{METHOD}

\section{Participants}

The sample consisted of 150 participants taken from the study by Rammsayer et al. (2017) and 90 newly recruited participants. Participants were 113 male and 127 female volunteers ranging in age from 17 to 32 years $\left(M_{\text {age }}=22.01\right.$ and $S D= \pm 3.02$ years). Recruiting took place within and outside the University of Bern, resulting in 136 university students and 104 individuals with a nonacademic background. All participants had normal or corrected-to-normal vision and were native German speakers. For taking part in the study, university students could choose between course credits or CHF 45.00; non-student participants received CHF 45.00. The study was approved by the local ethics committee and all participants gave their written informed consent.

\section{Measurement of Psychometric Intelligence}

A modified short version of the BIS test (Jäger et al., 1997) was administered. The BIS test is based on Jäger's (1984) BIS model of intelligence, which classifies cognitive abilities along two dimensions: (a) the mental operation required to solve a subtest and (b) the content of a given subtest. In the present study, 18 BIS subtests were used to measure processing speed (BIS-Speed), processing capacity (BIS-Capacity), and memory (BIS-Memory) as three major factors of psychometric intelligence. Each factor was assessed by two figural, two numeric, and two verbal subtests. In a previous pilot study with a sample of 122 participants, Wicki (2014) showed satisfactory test-retest reliabilities $\left(r_{\mathrm{tt}}\right)$ and composite reliabilities as measured by McDonald's (1999) omega $(\Omega)$ for all three factors of intelligence obtained with the very same subtests as in the present study: $r_{\mathrm{tt}}=.85, \Omega=.58$ for BIS-Speed, $r_{\mathrm{tt}}=.64$ and $\Omega=$ .79 for BIS-Capacity, and $r_{\text {tt }}=.86, \Omega=.63$ for BIS-Memory.

The individual performance score (i.e., the number of correctly solved items) of each subtest was $z$-standardized. The manifest correlations are based on BIS operation mean scores (i.e., all six $z$-standardized subtests scores of the same operation were aggregated to a mean score). For the latent analysis, $z$-standardized test scores on the two subtests assessing the same operation with the same content were averaged. Afterwards, latent variables reflecting BIS-Speed, BIS-Capacity, and BIS-Memory were derived from the respective verbal, numeric, and figural average scores.

\section{Hick Reaction Time Paradigm}

For the experimental assessment of SIP, a Hick reaction time paradigm was used similar to the one proposed by Rammsayer and Brandler (2007).

\section{Apparatus and Stimuli}

The presentation of stimuli was controlled by E-Prime 2.0 software running on a Dell Optiplex 760 Computer with an 18" Samsung SyncMaster 900SL monitor. Stimuli were white (RGB color coordinates: $255,255,255)$ rectangles $(1.6 \mathrm{~cm} \times 1.4 \mathrm{~cm})$ and plus signs $(0.5$ $\mathrm{cm})$ presented on a black $(0,0,0)$ monitor screen. For registration of the participant's responses, a Cedrus response pad (Model RB-830; Cedrus Corporation; n.d.) was used with four buttons corresponding to the locations of the four rectangles presented under the 2-bit condition (see Figure 1). Responses were recorded with an accuracy of \pm 1 ms.

\section{Procedure}

In the 0-bit condition (no-choice or simple reaction time), each trial started with the presentation of a rectangle in the center of the monitor screen. After a foreperiod varying randomly between 1 and $2 \mathrm{~s}$ in steps of $333 \mathrm{~ms}$, the imperative stimulus, a plus sign, was presented in the center of the rectangle (see Figure 1). The rectangle and the plus sign remained on the screen until the participant pressed a designated response button. The 1-bit condition (two-choice reaction time) was almost identical to the 0 -bit condition, except that two rectangles were 


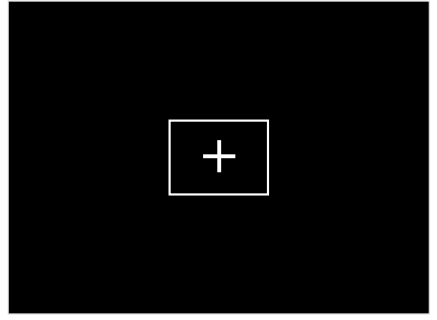

0-bit condition

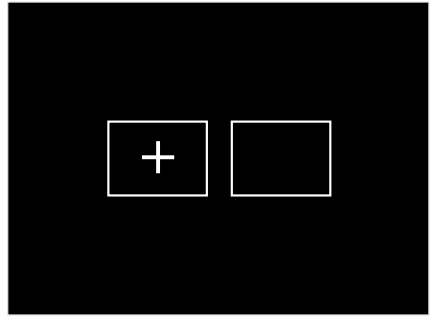

1-bit condition

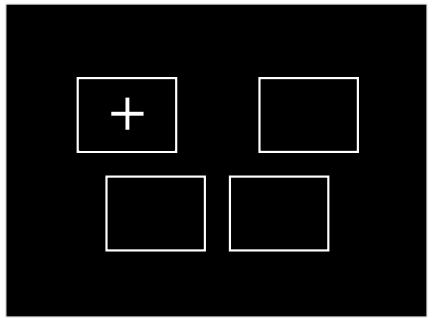

2-bit condition

FIGURE 1.

Stimulus presentation for the three Hick conditions.

presented arranged in a row. After a variable foreperiod, the imperative stimulus was presented in one of the two rectangles. Presentation of the imperative stimulus was randomized and balanced. Thus, the imperative stimulus appeared in each of the two rectangles in $50 \%$ of the trials. Similarly, in the 2-bit condition (four-choice reaction time) four rectangles arranged in two rows were displayed on the monitor screen. Again, the imperative stimulus was presented randomly in one of the four rectangles after a variable foreperiod.

The instruction to the participants emphasized responding as quickly as possible to the imperative stimulus by pressing the response key corresponding to the rectangle with the imperative stimulus but to avoid response errors. After an intertrial interval of 1,100 ms, the next trial started. Incorrect responses were followed by a 200 -ms tone. Hick conditions were presented in ascending order (cf. Jensen, 2006). Each condition consisted of 32 trials preceded by 10 practice trials. As an indicator of individual SIP, mean reaction time was computed separately for the 0-, 1-, and 2-bit conditions.

\section{Statistical Analyses}

All statistical analyses were performed in $\mathrm{R}$ (Version 3.3.0; R Core Team, 2015) using the lavaan package (Rosseel, 2012) for confirmatory factor analysis and structural equation modeling. Since the Hick reaction time data were not normally distributed, the Satorra-Bentler robust maximum likelihood estimation method (Satorra \& Bentler, 1994) was used. The model fit was evaluated with the Satorra-Bentler adjusted chi-square $\left(\chi^{2}\right)$ test statistic, as well as with the following fit indices: $\chi^{2} / d f$-ratio, comparative fit index (CFI), root mean square error of approximation (RMSEA), and standardized root mean square residual (SRMR). A $\chi^{2} / d f$-ratio smaller than 2 (Carmines \& McIver, 1983), CFI $\geq .95$ (Hu \& Bentler, 1999), an RMSEA $\leq .08$ (Browne \& Cudeck, 1993), and an SRMR $\leq .08$ (Hu \& Bentler, 1999) are considered indicators of a good model/data fit. Competing models were compared by means of the Satorra-Bentler adjusted $\chi^{2}$-difference test (Satorra \& Bentler, 2001) as well as the Akaike information criterion (AIC). A model with a lower AIC is considered the more parsimonious model (Kline, 2011). More detailed information on the applied fit indices is provided by $\mathrm{Hu}$ and Bentler (1999) and Schermelleh-Engel, Moosbrugger, and Müller (2003).

\section{RESULTS}

Descriptive statistics of scores on the 18 BIS subtests and reaction time performance of the Hick task are given in Table 1 . As indicated by $t$ tests, mean Hick reaction time increased significantly from the 0 - to the 1-bit condition, $t(239)=-37.65, p<.001, d=2.57$, and from the 1 - to the 2 -bit condition, $t(239)=-32.01, p<.001, d=2.37$, underscoring that the experimental manipulation was successful. Correlations among the manifest intelligence mean scores and Hick reaction times were statistically significant, except for the correlations between reaction times in the three Hick conditions and memory (Table 2).

\section{Conventional Confirmatory Factor Analysis of Hick Reaction Time Data and Psychometric Intelligence}

The measurement model of intelligence was based on nine manifest variables, with scores from subtests assessing the same operation with the same content being averaged, respectively. From the three average scores of the same operation, latent variables representing BIS-Speed, BIS-Capacity, and BIS-Memory were derived. The three latent variables as well as the residual variances of the same content were allowed to correlate with each other. The resulting model showed a good fit, $\chi^{2}(15)=25.235, p<.05, \mathrm{CFI}=.987, \mathrm{RMSEA}=.053$, SRMR $=.033$. The congeneric measurement model of the Hick data was not inspected separately since just identified models with zero degrees of freedom yield a trivially perfect fit (Kline, 2011).

Subsequently, the congeneric measurement model of the Hick reaction time data was related to BIS-Speed, BIS-Capacity, and BISMemory (see Figure 2). This model yielded a good fit $\chi^{2}(39)=63.845, p$ $<.01, \mathrm{CFI}=.979, \mathrm{RMSEA}=.052, \mathrm{SRMR}=.046$. The latent variable derived from the three mean reaction times in the Hick task significantly predicted BIS-Speed, $\beta=-.352, p<.001$, BIS-Capacity, $\beta=-.230, p<$ .001 , and BIS-Memory, $\beta=-.151, p<.05$. Although it seems as if the relationship between Hick reaction time and BIS-Speed was stronger than the relationship between Hick reaction time and BIS-Capacity as well as between Hick reaction time and BIS-Memory, these three 


\begin{tabular}{|c|c|c|c|c|c|c|}
\hline Performance measure & Mean & $S D$ & $\min$ & $\operatorname{Max}$ & Operation & Content \\
\hline \multicolumn{7}{|l|}{ BIS subtests } \\
\hline City map & 15.3 & 4.4 & 4 & 26 & M & $\mathrm{F}$ \\
\hline Number sequences & 4.0 & 2.6 & 0 & 9 & $\mathrm{C}$ & $\mathrm{N}$ \\
\hline Figural analogies & 3.3 & 1.6 & 0 & 8 & $\mathrm{C}$ & $\mathrm{F}$ \\
\hline $\mathrm{X}$-larger & 19.8 & 8.1 & 1 & 44 & $S$ & $\mathrm{~N}$ \\
\hline Verbal analogies & 3.5 & 2.0 & 0 & 8 & $\mathrm{C}$ & $\mathrm{V}$ \\
\hline Paired associates & 6.0 & 2.3 & 0 & 12 & M & $\mathrm{N}$ \\
\hline Fact-opinion & 9.3 & 3.6 & 2 & 16 & $\mathrm{C}$ & $\mathrm{V}$ \\
\hline Crossing letters & 53.5 & 9.1 & 28 & 82 & S & $\mathrm{F}$ \\
\hline Estimation & 3.5 & 1.9 & 0 & 7 & $\mathrm{C}$ & $\mathrm{N}$ \\
\hline Story & 8.3 & 3.5 & 1 & 20 & M & V \\
\hline Charkow & 3.0 & 1.7 & 0 & 6 & $\mathrm{C}$ & $\mathrm{F}$ \\
\hline Part-whole & 11.4 & 3.2 & 1 & 20 & S & $\mathrm{V}$ \\
\hline Math operators & 10.0 & 4.0 & 1 & 20 & S & $\mathrm{N}$ \\
\hline Word memory & 7.0 & 2.6 & 1 & 17 & M & V \\
\hline Word classification & 22.9 & 6.2 & 1 & 36 & $S$ & V \\
\hline Two-digit numbers & 6.8 & 2.8 & 0 & 19 & M & $\mathrm{N}$ \\
\hline Old English & 32.2 & 6.0 & 4 & 48 & $S$ & $\mathrm{~F}$ \\
\hline Routes memory & 18.7 & 5.6 & 1 & 31 & M & $\mathrm{F}$ \\
\hline \multicolumn{7}{|l|}{ Hick task conditions } \\
\hline 0-bit & 251 & 30 & 190 & 373 & & \\
\hline 1-bit & 307 & 33 & 243 & 448 & & \\
\hline 2-bit & 380 & 51 & 263 & 624 & & \\
\hline
\end{tabular}

Note. Descriptive statistics for the 18 Berlin Intelligence Structure (BIS) subtests (listed in order of presentation) based on raw scores before $z$-standardization as well as for reaction times in milliseconds of the 0-, 1-, and 2-bit condition of the Hick task. Also given are the mental operation and the content of each BIS subtest. $\mathrm{C}=$ processing capacity; $\mathrm{S}=$ processing speed; $\mathrm{M}=$ memory; $\mathrm{F}=$ figural; $\mathrm{V}=$ verbal; $\mathrm{N}=$ numeric.

\section{TABLE 2.}

Correlations Among the Manifest Intelligence Variables of Processing Speed (BIS-Speed), Processing Capacity (BIS-Capacity), Memory (BIS-Memory), and Mean Reaction Times of the Three Hick Task Conditions

\begin{tabular}{|c|c|c|c|c|c|}
\hline & BIS-Speed & BIS-Capacity & BIS-Memory & 0-bit & 1-bit \\
\hline BIS-Capacity & $.59^{\star * \star}$ & & & & \\
\hline BIS-Memory & $.54^{\star * \star}$ & $.52^{\star * *}$ & & & \\
\hline 0-bit & $-.29^{* * *}$ & $-.16^{*}$ & -.08 & & \\
\hline 1-bit & $-.30^{\star * *}$ & $-.13^{\star}$ & -.10 & $.74^{\star * *}$ & \\
\hline 2-bit & $-.32^{* * *}$ & $-.25^{\star * *}$ & -.12 & $.62^{* * *}$ & $.73^{\star * *}$ \\
\hline
\end{tabular}

Note. BIS-Speed = Processing Speed, BIS-Capacity $=$ Processing Capacity, BIS-Memory $=$ Memory, BIS $=$ Berlin Intelligence Structure Test; ${ }^{*} p<.05 ;{ }^{* * *} p<.001$ (two tailed).

$\beta$ coefficients did not differ statistically from each other. This was revealed when a constrained model with all three regression coefficients set to be equal was compared to the unconstrained model. The fit of the constrained model, $\chi^{2}(41)=66.434, p<.01, \mathrm{CFI}=.978$, RMSEA $=$ .051 , SRMR $=.049$, was not significantly worse than the fit of the un- constrained model, as indicated by a $\chi^{2}$-difference test, $\Delta \chi^{2}(2)=2.459$, $p=.292$. Moreover, the constrained model, AIC $=8315.045$, was more parsimonious than the unconstrained model, AIC $=8316.939$. These results indicate that Hick predicted the three aspects of psychometric intelligence equally well. 


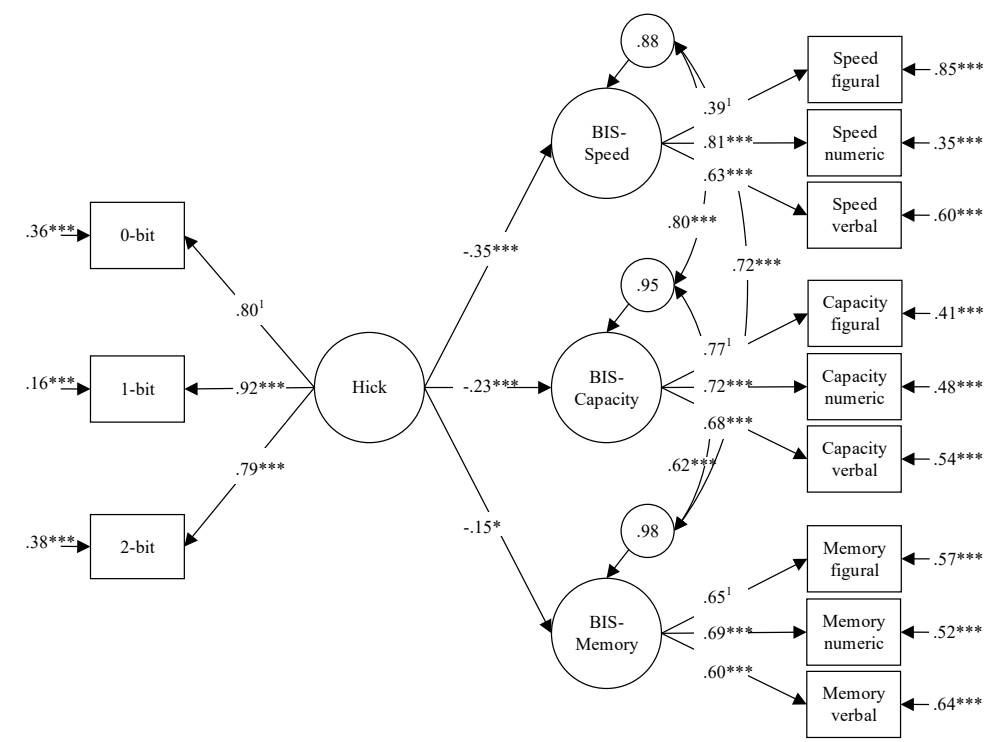

FIGURE 2.

The congeneric Hick measurement model based on the mean reaction times of the three Hick task conditions (0-, 1-, and 2-bit) related to the Berlin Intelligence Structure (BIS) measurement model with the three operations' processing speed (BIS-Speed), processing capacity (BIS-Capacity), and memory (BIS-Memory). ${ }^{*} p<.05 * * * 0.001$ (two-tailed).

\section{Fixed-links Models of Hick Reaction Time Data and Psychometric Intelligence}

In the next step, the Hick data was analyzed by means of fixed-links modeling to examine whether two independent latent variables describe the variance and covariance matrix of reaction time in the three Hick conditions appropriately. For the fixed-links model, the unstandardized factor loadings of the nonexperimental latent variable were all fixed to 1 , whereas the experimental latent variable had increasing factor loadings across the three Hick conditions. Since the number of response alternatives systematically increased across Hick conditions, we used a monotonically increasing function (i.e., 1, 2, 4) corresponding to the increasing number of possible responses (see Figure 3 ). The fixed-links model showed a good fit, $\chi^{2}(1)=0.305, p=.580$, CFI $=$ $1.000, \mathrm{RMSEA}=.000, \mathrm{SRMR}=.013$, and the variance of both latent variables was significant $(p<.001)$. In addition, the scaling of variances showed that $50.3 \%$ of latent variance was explained by the experimental latent variable and $49.7 \%$ - by the nonexperimental latent variable (for the procedure of scaling see Schweizer, 2011).

As in Rammsayer et al. (2017), the experimental and the nonexperimental latent variable were related to a higher-order $g$ factor of intelligence based on the three lower-level BIS operations. This model yielded a good fit, $\chi^{2}(41)=63.549, p<.05, \mathrm{CFI}=.981$, RMSEA $=.049$, SRMR $=.049$, and the experimental, $\beta=-.328, p<.001$, as well as the nonexperimental latent variable, $\beta=-.169, p<.05$, significantly predicted g. Subsequently, the fixed-links model of the Hick data was related to all three BIS operations (see Figure 4). This model yielded a good fit, $\chi^{2}(37)=56.388, p<.05$, CFI $=.983$, RMSEA $=.047$, SRMR $=.043$. The experimental latent variable significantly predicted BISSpeed, $\beta=-.314, p<.05$, and BIS-Capacity, $\beta=-.346, p<.01$, whereas no significant prediction was shown in relation to BIS-Memory, $\beta=$ $-.170, p=.075$. The nonexperimental latent variable significantly predicted BIS-Speed, $\beta=-.219, p<.05$, whereas no significant prediction was shown in relation to BIS-Capacity, $\beta=-.047, p=.548$, or BISMemory, $\beta=-.060, p=.532$. The experimental and nonexperimental latent variable combined explained the following portions of variance in the respective BIS operation: $14.7 \%$ in BIS-Speed, $12.2 \%$ in BISCapacity, and 3.3\% in BIS-Memory.

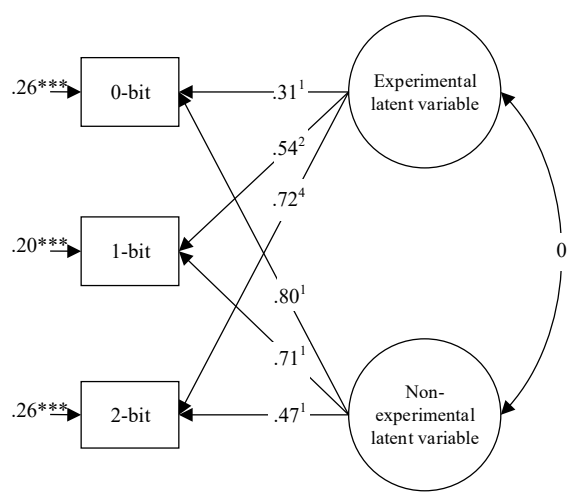

\section{FIGURE 3.}

Fixed-links measurement model decomposing reaction time variance of the Hick task into two independent latent variables: an experimental and a nonexperimental one. The factor loadings of the nonexperimental latent variable are fixed to 1 , whereas factor loadings of the experimental latent variable are fixed to the number of possible responses (i.e., 1, 2, and 4) in each Hick task condition. The superscript numbers denote the unstandardized fixed factor loadings. *** $p<.001$ (two-tailed). 


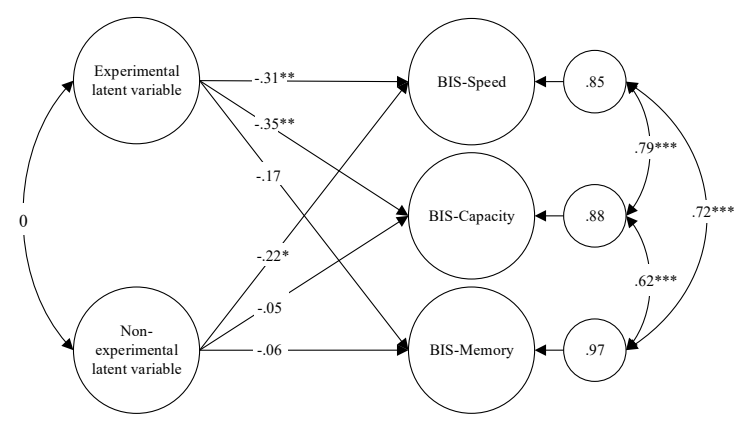

FIGURE 4.

The structural model relating the experimental and the nonexperimental latent variable to processing speed (BISSpeed), processing capacity (BIS-Capacity), and memory (BIS-Memory). ${ }^{*} p<.05^{* *} p<.01{ }^{* * *} p<.001$ (two-tailed).

Equality constraints were applied to test whether the experimental latent variable was a better predictor of BIS-Capacity than BISSpeed as well as BIS-Memory. When the unstandardized regression coefficients from the experimental latent variable to BIS-Capacity, $b$ $=-.252$, and to BIS-Speed, $b=-.116$, were constrained to be equal, the resulting model fit was significantly worse compared to the unconstrained model, $\Delta \chi^{2}(1)=4.438, p<.05$. The same was true when the unstandardized regression coefficients from the experimental latent variable to BIS-Capacity and to BIS-Memory, $b=-.101$, were constrained to be equal, $\Delta \chi^{2}(1)=4.341, p<.05$. However, the model constraining the coefficients between the experimental latent variable to BIS-Speed and BIS-Memory to be equal did not show a worse fit than the unconstrained model, $\Delta \chi^{2}(1)=.075, p=.785$. In addition, this last-mentioned constrained model, AIC $=8312.048$, was shown to be more parsimonious than the unconstrained model, AIC $=8314.349$. Consequently, the path from the experimental latent variable to BISCapacity was significantly stronger than the two other paths, which did not differ significantly from each other.

Furthermore, we tested whether the nonexperimental latent variable exclusively predicted BIS-Speed rather than all three BIS operations (see Figure 4). For this purpose, all three $b$ coefficients of the nonexperimental latent variable were set to be equal. Surprisingly, the constrained model did not show a significantly worse fit than the unconstrained model, $\Delta \chi^{2}(2)=1.308, p=.520$. In fact, the constrained model, $\mathrm{AIC}=8311.444$, turned out to be more parsimonious than the unconstrained model, AIC $=8314.349$. In the light of the weak but statistically significant association between the nonexperimental latent variable and BIS-Speed, this latter finding indicated that the observed functional relationships between the nonexperimental latent variable and each of the three BIS operations can be considered equally strong.

\section{DISCUSSION}

In the present study, the major goal was to arrive at a better understanding of the relationship between SIP (as measured with the Hick paradigm) and speed-, capacity-, and memory-related aspects of psychometric intelligence (as measured with the BIS test). For this purpose, fixed-links modeling was applied to dissociate SIP into two sources of variance referred to as experimental and nonexperimental latent variable. On the one hand, the experimental latent variable represented reaction time variance caused by individual differences in the response selection process directly affected by the systematic increase in the number of possible responses across Hick task conditions. On the other hand, the nonexperimental latent variable reflected variance caused by residual sources unaffected by the experimental manipulation of task demands (e.g., sensorimotor speed or subjective mental/ physical states). We found the strongest relationship between the experimental latent variable and BIS-Capacity and a weaker but still substantial relationship between the experimental latent variable and BIS-Speed. The nonexperimental latent variable showed only a weak relationship to BIS-Speed. Additionally, in contrast to Rammsayer et al. (2017), we found that not only the experimental but also the nonexperimental latent variable was substantially related to $g$.

At the manifest level, the correlations between Hick reaction time and the three aspects of psychometric intelligence were only moderate, as also reported previously (Jensen, 1987; Sheppard \& Vernon, 2008). Furthermore, these correlations showed a similar relational precedence and magnitude as those reported by Beauducel and Brocke (1993). Hick reaction times were moderately related to BIS-Speed and to a lesser extent, but still significantly, to BIS-Capacity. In line with previous research, the more demanding Hick task conditions affording higher task demands exhibited stronger correlations with intelligence than the less demanding Hick task conditions (Rammsayer \& Troche, 2016; Stankov, 2000; Vernon \& Weese, 1993). According to Jensen $(2006,2011)$, more demanding Hick conditions require to deal with an increased information load, and thus, should show a stronger functional relationship with psychometric intelligence. In contrast to Beauducel and Brocke (1993), no substantial associations were found between Hick reaction times and memory. A possible explanation for this finding is that memory processes are only marginally involved in solving the Hick task.

At the latent level, conventional confirmatory factor analysis was used to derive a general SIP factor from reaction time in the three Hick task conditions. Descriptively, this rather impure SIP factor was more closely related to BIS-Speed than to BIS-Capacity and BIS-Memory. Similar results were reported by Beauducel and Brocke (1993) as well as by Neubauer and Bucik (1996). As revealed by significance testing, however, the impure SIP factor predicted all three aspects of intelligence to an equivalent degree. Also, Helmbold and Rammsayer (2006) reported that a latent general Hick factor showed virtually identical correlations to speed- and capacity-related aspects of intelligence. Thus, it seems that the different sources of reaction time variance comprised in a congeneric SIP factor might account for its undifferentiated relationship with these various aspects of psychometric intelligence.

As a more adequate approach to impure SIP than conventional confirmatory factor analysis, fixed-links modeling was applied to decompose reaction time variance into two functionally independent sources of variance referred to as experimental and nonexperimental 
latent variable, respectively. It is important to note that our experimental latent variable provides a qualitatively different measure than the traditional slope parameter proposed by Hick (1952). The slope parameter indicates the linear increase in response time with the logarithm of the number of choice alternatives and still suffers from insufficient purity. However, the experimental latent variable derived in the present study can be considered a purer measure reflecting the portion of variance in SIP solely due to the systematic increase in the number of possible responses across the three Hick task conditions. From this perspective, our experimental latent variable represents reaction time variance originating from the stage of response selection.

The time taken by the response selection process(es) related to the increased number of possible responses across Hick task conditions has been assumed to be an indicator of an individual's maximum rate of information processing (Leite \& Ratcliff, 2010; Logan, van Zandt, Verbruggen, \& Wagenmakers, 2014; Schneider \& Anderson, 2011; Usher \& McClelland, 2001). From this point of view, the experimental latent variable can be understood as a measure of the capacity for processing information, which is consistent with our finding of a substantial relation to BIS-Capacity.

In contrast, our nonexperimental latent variable represents a conglomerate of various speed-related sources. These sources remained constantly effective across the three Hick conditions and, thus, caused individual differences in reaction time independently of the experimental manipulation. These sources of constant influence might comprise basic processing speed (e.g., Heitz, Unsworth, \& Engle, 2005), basal speed of sensorimotor processing (e.g., Jensen, 2006; Schweizer, 2007), or also subjective state variables such as the motivation to perform or current levels of individual fatigue (e.g., Thomas et al., 2015). The obtained fixed-links model fit the data well. Hence, the dissociation of experimental from nonexperimental reaction time variance could be considered successful.

As in previous research (Rammsayer et al., 2017), the experimental latent variable predicted a larger portion of variance in psychometric $g$ than the nonexperimental latent variable. This stronger association is consistent with the common finding that the $g$ factor of intelligence is positively related to increasing task demands (e.g., Rammsayer \& Troche, 2016; Stankov, 2000; Vernon, \& Weese, 1993). In contrast to Rammsayer et al.s (2017) previous finding of a nonsignificant association between the nonexperimental latent variable and psychometric $\mathrm{g}$, this relationship became statistically significant with the larger sample of the present study. Thus, individual differences in the residual sources of reaction time that are considered constant across task conditions and are thus unaffected by the systematic experimental manipulation, seemed to also account for a small portion of variance in psychometric $g$.

At the descriptive level, the experimental latent variable showed a moderate association with BIS-Speed and BIS-Capacity but no substantial association with BIS-Memory. At the statistical level, however, SIP related to the increasing Hick task demands was a better predictor of BIS-Capacity than BIS-Speed. In that regard, our data indicated that speed of processing induced by increased task demands (i.e., more possible responses across task conditions) accounted much stronger for individual differences in capacity- than for speed-related aspects of intelligence. This finding is consistent with the notion that the experimental latent variable represented variance originating from the stage of response selection and can be considered as a measure of capacity for processing information (see above). Another possible reason for this pattern of results can be seen in the two different types of tests used in the psychometric assessment of intelligence: power and speed tests. Power tests are characterized by increasing task difficulty across items and allow sufficient time for an individual to answer an item. Hence, an individual's number of correctly solved items depends on her/his ability to cope with the increasing task demands rather than on the speed with which the items were solved (cf. Jensen 1980; Murphy \& Davidshofer, 2005). Unlike power tests, speed tests are characterized by trivially easy items but high time constraints (Murphy \& Davidshofer, 2005). Therefore, an individual's number of correctly solved items primarily depends on her/his response speed and only to a very small degree on her/his cognitive processing capacity.

With the BIS test, BIS-Capacity is measured by power tests with items arranged in order of ascending difficulty and-despite a time limit-sufficient time is given to solve these items. Previous research on this issue, however, showed that the relationship between SIP and psychometric intelligence remained substantial even after controlling for the influence of time-limited testing (Vernon \& Kantor, 1986; Wilhelm \& Schulze, 2002). Furthermore, the BIS-Memory subtests in the present study were also applied with a time-limitation, but there was no indication of a significant relationship with the experimental latent variable. Based on these findings, it is highly unlikely that the time-limited application of the BIS-Capacity subtests was the actual cause for the observed relationship between processing capacity and SIP as reflected by the experimental latent variable. Rather, our results are in line with Jensen's (2006) assumption that when too much information has to be processed concurrently, the capacity-limited cognitive systems involved in the processing of a given test item are prone to overload resulting in a breakdown of the system. Faster information processing (as indicated by, e.g., shorter reaction time in the Hick task) facilitates the processing of more information without overstraining the limited resources of the system. From this point of view, it is plausible that in particular the experimental latent variable showed a substantial relationship with BIS-Capacity, as this latent variable represents the speed of processing associated with the increasing task demands.

Against this background, it is plausible that the experimental latent variable, representing capacity of information processing, showed the strongest functional relationship with the cognitively more demanding capacity-related aspect of intelligence. Also the relationship between the experimental latent variable and the speed-related aspect of intelligence was significant. This finding can be explained by the fact that although the BIS-Speed subtests induced only very low task demands, these items still required cognitive processing capacities, albeit at a lower level.

With regard to the nonexperimental latent variable, the strongest association was observed in relation to the speed-related aspect of in- 
telligence. At the statistical level, however, the generally low strength of the relationships between the nonexperimental latent variable and all three BIS operations did not differ significantly from each other. As a possible reason, the nonexperimental latent variable may represent variance that is equally related to all three aspects of intelligence. For example, subjective states like the motivation to perform or a lack of concentration due to fatigue could influence performance in each aspect of intelligence to about the same degree.

The relationship between all speed variables derived from the Hick task (i.e., the congeneric Hick factor and the two dissociated fixed-links components) and BIS-Speed were quite low, indicating some shared variance but not identity. Hence, the Hick task might not reflect the speed-related aspect of intelligence very well. However, these findings are in line with contemporary structural models of intelligence such as the Cattell-Horn-Carroll model, which differentiates between cognitive processing speed and decision/reaction time or speed (Carroll, 1993; McGrew, 2005). The former refers to the ability "to automatically and fluently perform relatively easy or overlearned cognitive tasks" (McGrew, 2005, p. 155), whereas the latter refers to the ability "to react and/or make decisions quickly in response to simple stimuli, typically measured by chronometric measures of reaction and inspection time" (McGrew, 2005, p. 155). Therefore, the weak relationships between the different speed variables derived from the Hick task and BIS-Speed supports the view of two different speed factors derived from speed tests used to represent BIS-Speed and from the reaction time data as used in the Hick task.

To sum up, the present study successfully dissociated reaction time variance into two functionally independent speed components. One component, representing individual differences in SIP directly related to the systematically increased number of possible responses, was most strongly linked with the capacity-related aspect of intelligence. The other component comprised individual differences in SIP unrelated to the experimental manipulation of response alternatives. Individual differences in SIP caused by experimentally increasing the number of possible responses were shown to be more strongly associated with capacity- than with speed-related aspects of psychometric intelligence. On the other hand, individual differences in SIP not associated with the experimental manipulation of response alternatives showed rather weak relationships to each of the three BIS operations and could be considered equally strong

\section{AUTHOR NOTE}

This work was supported by the Swiss National Science Foundation (Grant No. 100014_146034).

\section{REFERENCES}

Alluisi, E. A., \& Warm, J. S. (1990). Things that go together: A review of stimulus-response compatibility and related effects. In R. W. Proctor \& T. G. Reeve (Eds.), Stimulus-response compatibility: An integrated perspective (pp. 3-30). Amsterdam, The Netherlands: Elsevier.

Beauducel, A., \& Brocke, B. (1993). Intelligence and speed of information processing: Further results and questions on Hick's paradigm and beyond. Personality and Individual Differences, 15, 627-636. doi: 10.1016/0191-8869(93)90004-M

Bors, D. A., \& Forrin, B. (1995). Age, speed of information processing, recall, and fluid intelligence. Intelligence, 20, 229-248. doi: 10.1016/0160-2896(95)90009-8

Browne, M. W., \& Cudeck, R. (1993). Alternative ways of assessing model fit. In K. A. Bollen \& J. S. Long (Eds.), Testing structural equation models (pp. 136-162). Newbury Park, CA: Sage.

Carmines, E. G., \& Mclver, J. P. (1983). An introduction to the analysis of models with unobserved variables. Political Methodology, 9, 51-102.

Carroll, J. B. (1993). Human cognitive abilities: A survey of factor analytic studies. Cambridge, England: Cambridge University Press.

Cedrus Model RB-830 [response pad]. (n.d.). San Pedro, CA: Cedrus Corporation.

Heitz, R. P., Unsworth, N., \& Engle, R. W. (2005). Working memory capacity, attention control, and fluid intelligence. In O. Wilhelm \& R. W. Engle (Eds.), Handbook of understanding and measuring intelligence (pp. 61-77). Thousand Oaks, CA: Sage.

Helmbold, N., \& Rammsayer, T. H. (2006). Timing performance as a predictor of psychometric intelligence as measured by speed and power tests. Journal of Individual Differences, 27, 20-37. doi: 10.1027/1614-0001.27.1.20

Hick, W. E. (1952). On the rate of gain of information. Quarterly Journal of Experimental Psychology, 4, 11-26. doi: 10.1080/17470215208416600

Hu, L., \& Bentler, P. M. (1999). Cutoff criteria for fit indexes in covariance structure analysis: Conventional criteria versus new alternatives. Structural Equation Modeling, 6, 1-55. doi: 10.1080/10705519909540118

Humphreys, M. S., \& Revelle, W. (1984). Personality, motivation, and performance: $A$ theory of the relationship between individual differences and information processing. Psychological Review, 91, 153-184. doi: 10.1037/0033-295X.91.2.153 |wWw

Jäger, A. O. (1984). Intelligenzstrukturforschung: Konkurrierende Modelle, neue Entwicklungen, Perspektiven [Intelligence structure research: Competing models, new developments, perspectives]. Psychologische Rundschau, 35, 21-35.

Jäger, A. O., Süss, H. M., \& Beauducel, A. (1997). Berliner Intelligenzstrukturtest. BIS-Test, Form 4 [Berlin Test of Intelligence Structure. BIS-Test, Form 4]. Göttingen, Germany: Hogrefe.

Jensen, A.R. (1980). Bias in mental testing. New York, NY: Free Press.

Jensen, A. R. (1987). Individual differences in the Hick paradigm. In P. A. Vernon (Ed.), Speed of information-processing and intelligence (pp. 101-175). Norwood, NJ: Ablex.

Jensen, A. R. (1998a). The g factor. Westport, CT: Praeger.

Jensen, A. R. (1998b). The suppressed relationship between IQ and the reaction time slope parameter of the Hick function. Intelligence, 26, 43-52. doi: 10.1016/S0160-2896(99)80051-8

Jensen, A. R. (2006). Clocking the mind: Mental chronometry and individual differences. Amsterdam, The Netherlands: Elsevier. 
Jensen, A. R. (2011). The theory of intelligence and its measurement. Intelligence, 39, 171-177. doi: 10.1016/j. intell.2011.03.004

Jensen, A. R., \& Munro, E. (1979). Reaction time, movement time, and intelligence. Intelligence, 3, 121-126. doi: 10.1016/01602896(79)90010-2

Kline, R. B. (2011). Principles and practice of structural equation modeling. New York, NY: Guilford Press.

Langner, R., Steinborn, M. B., Chatterjee, A., Sturm, W., \& Willmes, K. (2010). Mental fatigue and temporal preparation in simple reaction-time performance. Acta Psychologica, 133, 64-72. doi: 10.1016/j.actpsy.2009.10.001 [wWW

Leite, F. P., \& Ratcliff, R. (2010). Modeling reaction time and accuracy of multiple-alternative decisions. Attention, Perception, \& Psychophysics, 72, 246-273. doi: 10.3758/APP.72.1.246 wwW

Lisper, H. O., \& Kjellberg, A. (1972). Effects of 24-hour sleep deprivation on rate of decrement in a 10-minute auditory reaction time task. Journal of Experimental Psychology, 96, 287-290. doi: 10.1037/h0033615 WwW

Logan, G. D., Van Zandt, T., Verbruggen, F., \& Wagenmakers, E.-J. (2014). On the ability to inhibit thought and action: General and special theories of an act of control. Psychological Review, 121, 66-95. doi: 10.1037/a0035230 WwW

Luce, D. (1986). Response times: Their role in inferring elementary mental organization. New York, NY: Oxford University Press.

McDonald, R. P. (1999). Test theory: A unified treatment. Mahwah, $\mathrm{NJ}$ : Erlbaum.

McGrew, K. S. (2005). The Cattell-Horn-Carroll theory of cognitive abilities. In D. P. Flanagan \& P. L. Harrison (Eds.), Contemporary intellectual assessment-Theories, tests, and issues (pp. 136181). New York, NY: Guilford Press.

Miller, J., \& Ulrich, R. (2013). Mental chronometry and individual differences: Modeling reliabilities and correlations of reaction time means and effect sizes. Psychonomic Bulletin \& Review, 20, 819-858. doi: 10.3758/s13423-013-0404-5 WWW

Murphy, K. R., \& Davidshofer, C. O. (2005). Psychological testing: Principles and applications. Boston, MA: Pearson.

Neubauer, A. C., \& Bucik, V. (1996). The mental speed-IQ relationship: Unitary or modular? Intelligence, 22, 23-48. doi: 10.1016/ S0160-2896(96)90019-7

Rammsayer, T. H., \& Brandler, S. (2007). Performance on temporal information processing as an index of general intelligence. Intelligence, 35, 123-139. doi: 10.1016/j.intell.2006.04.007

Rammsayer, T. H., Pahud, O., \& Troche, S. J. (2017). Decomposing the functional relationship between speed of information processing in the Hick paradigm and mental ability: A fixedlinks modeling approach. Personality and Individual Differences, 118, 17-21. doi: 10.1016/j.paid.2017.01.050

Rammsayer, T. H., \& Troche, S. J. (2016). Validity of the worst performance rule as a function of task complexity and psychometric g: On the crucial role of g saturation. Journal of Intelligence, 4:5. doi:10.3390/jintelligence4010005
R Core Team [Computer software] (2015). Retrieved from https:// www.R-project.org/

Ren, X., Schweizer, K., \& Xu, F. (2013). The sources of the relationship between sustained attention and reasoning. Intelligence, 41, 51-58. doi: 10.1016/j.intell.2012.10.006

Rosseel, Y. (2012). lavaan: An R package for structural equation modeling. Journal of Statistical Software, 48, 1-36.

Satorra, A., \& Bentler, P. M. (1994). Corrections to test statistics and standard errors in covariance structure analysis. In A. Eye \& C. C. Clogg (Eds.), Latent variable analysis: Applications to developmental research (pp. 399-419). Thousand Oaks, CA: Sage.

Satorra, A., \& Bentler, P. M. (2001). A scaled difference chi-square test statistic for moment structure analysis. Psychometrika, 66, 507-514. doi: 10.1007/BF02296192

Schermelleh-Engel, K., Moosbrugger, H., \& Müller, H. (2003). Evaluating the fit of structural equation models: Tests of significance and descriptive goodness-of-fit measures. Methods of Psychological Research Online, 8, 23-74.

Schneider, D. W., \& Anderson, J. R. (2011). A memory-based model of Hick's law. Cognitive Psychology, 62, 193-222. doi: 10.1016/j. cogpsych.2010.11.001 www

Schweizer, K. (2006). The fixed-links model for investigating the effects of general and specific processes on intelligence. Methodology, 2, 149-160. doi: 10.1027/1614-2241.2.4.149

Schweizer, K. (2007). Investigating the relationship of working memory tasks and fluid intelligence tests by means of the fixed-links model in considering the impurity problem. Intelligence, 35, 591-604. doi: 10.1016/j.intell.2006.11.004

Schweizer, K. (2008). Investigating experimental effects within the framework of structural equation modeling: An example with effects on both error scores and reaction times. Structural Equation Modeling, 15, 327-345. doi: 10.1080/10705510801922621

Schweizer, K. (2011). Scaling variances of latent variables by standardizing loadings: Applications to working memory and the position effect. Multivariate Behavioral Research, 46, 938-955. doi: 10.1080/00273171.2011.625312 WwW

Sheppard, L. D., \& Vernon, P. A. (2008). Intelligence and speed of information-processing: A review of 50 years of research. Personality and Individual Differences, 44, 535-551. doi: 10.1016/j.paid.2007.09.015

Stankov, L. (2000). Complexity, metacognition, and fluid intelligence. Intelligence, 28, 121-143. doi: 10.1016/S01602896(99)00033-1

Stauffer, C. C., Troche, S. J., Schweizer, K., \& Rammsayer, T. H. (2014). Intelligence is related to specific processes in visual change detection: Fixed-links modeling of hit rate and reaction time. Intelligence, 43, 8-20. doi: 10.1016/j.intell.2013.12.003

Sternberg, S. (1969). Memory-scanning: Mental processes revealed by reaction-time experiments. American Scientist, 57, 421-457. WWW

Tabachnick, B. G., \& Fidell, L. S. (2013). Using multivariate statistics. Boston, MA: Pearson. 
Thomas, P., Rammsayer, T. H., Schweizer, K., \& Troche, S. J. (2015). Elucidating the functional relationship between working memory capacity and psychometric intelligence: A fixed-links modeling approach for experimental repeated-measures designs. Advances in Cognitive Psychology, 11, 3-13. doi: 10.5709/ acp-0166-6 $\underline{\underline{W W}]}$

Troche, S. J., Schweizer, K., \& Rammsayer, T. H. (2009). The relationship between attentional blink and psychometric intelligence: A fixed-links model approach. Psychology Science Quarterly, 51, 432-448. doi: 10.1016/j.paid.2013.10.023

Unsworth, N., \& Engle, R. W. (2007). On the division of short-term and working memory: An examination of simple and complex span and their relation to higher order abilities. Psychological Bulletin, 133, 1038-1066. doi: 10.1037/0033-2909.133.6.1038 WWW

Usher, M., \& McClelland, J. L. (2001). The time course of perceptual choice:The leaky, competing accumulator model. Psychological Review, 108, 550-592. doi: 10.1037/0033-295X.108.3.550 [wWW]

Van Zomeren, A. H., \& Brouwer, W. H. (1994). Clinical neuropsychology of attention. New York, NY: Oxford University Press.

Vernon, P. A., \& Kantor, L. (1986). Reaction time correlations with intelligence test scores obtained under either timed or untimed conditions. Intelligence, 10, 315-330. doi: 10.1016/01602896(86)90002-4
Vernon, P. A., \& Weese, S. E. (1993). Predicting intelligence with multiple speed of information-processing tests. Personality and Individual Differences, 14, 413-419. doi: 10.1016/01918869(93)90310-Y

Wagner, F. L., Rammsayer, T. H., Schweizer, K., \& Troche, S. J. (2014). Relations between the attentional blink and aspects of psychometric intelligence: A fixed-links modeling approach. Personality and Individual Differences, 58, 122-127. doi: 10.1016/j.paid.2013.10.023

Wagner, F. L., Rammsayer, T. H., Schweizer, K., \& Troche, S. J. (2015). A fixed-links modeling approach to assess individual differences in the attentional blink: Analysis of behavioral and psychophysiological data. Acta Psychologica, 159, 123-130. doi: 10.1016/j.actpsy.2015.06.002 www

Wicki, J. (2014). Struktur- und Reliabilitätsanalyse einer modifizierten Kurzversion des Berliner Intelligenzstruktur-Tests [Structure and reliability analysis of a modified short version of the Berlin Intelligence Structure Test] (Unpublished master thesis). University of Bern, Switzerland.

Wilhelm, O., \& Schulze, R. (2002). The relation of speeded and unspeeded reasoning with mental speed. Intelligence, 30, 537-554. doi: 10.1016/S0160-2896(02)00086-7

RECEIVED 29.05.2017| ACCEPTED 30.11.2017 